\title{
Neutrino Point Source Search including Cascade Events with the ANTARES Neutrino Telescope
}

\author{
Tino Michael* on behalf of the ANTARES collaboration \\ Nikhef \\ E-mail: tino.michael@nikhef.nl
}

\begin{abstract}
ANTARES is the largest neutrino telescope in the Northern Hemisphere. It has been taking data since 2007. One of the prime objectives is the detection and identification of cosmic neutrino sources in the $\mathrm{TeV}$ to $\mathrm{PeV}$ energy regime. ANTARES has established excellent pointing resolution for muon neutrinos $(0.4 \mathrm{deg})$. Recently, we achieved good pointing capabilities also for contained cascade events $\left(\approx 2^{\circ}\right)$, which opens up the possibility for all-flavour neutrino point source searches. Together with its geographical location, this makes ANTARES an excellent/competitive tool to test for the presence of cosmic sources in the Southern Hemisphere, including the area around the Galactic Centre, where IceCube reports a slight excess.

In this contribution, we briefly discuss the method to measure the shower energy and direction, which yields degree-level resolutions. We also present the latest time-integrated point source search results, which incorporate cascade events alongside the muon-neutrino events, and the impact on the interpretation of the IceCube signal.
\end{abstract}

The 34th International Cosmic Ray Conference,

30 July- 6 August, 2015

The Hague, The Netherlands

\footnotetext{
* Speaker.
} 


\section{Introduction}

The ANTARES neutrino telescope has been operating in the Mediterranean sea since 2007. The clarity of the sea water allows for an excellent timing measurement of the Cherenkov light induced by charged particles, so that an angular resolution better than 0.4 degrees could be established for up-going muon neutrinos. This allows Antares to be competitive to IceCube in the search for point sources in the Southern Hemisphere despite its small size.[1]

Adding sensitivity to cascade events provides access to $v_{\mathrm{e}}$ charged current interactions (and from there, estimate $v_{\tau} \rightarrow \tau \rightarrow$ e contributions) and all flavour neutral current interactions and therefore increases the sensitivity for cosmic neutrino sources even further.

\section{Cascade Reconstruction}

Cascade events are reconstructed using a novel algorithm, which was developed for the purpose of point source searches. The reconstruction proceeds in two stages:

Assuming a spherically expanding shell of photons, the shower mean position (which is close to, but not equal to the neutrino interaction) and the time of occurrence are fitted using the detected photon arrival times. The optical background present in ANTARES is mitigated by the use of a robust so-called M-estimator ${ }^{1}$.

The shower direction is determined from the intensities of the detected Cherenkov light. While the timing structure of the light is spherical to a good approximation, cascade events cause most light to be emitted under the Cherenkov angle. The likelihood fit uses a tabulated probability density function (PDF) of the expected number of photons as a function of the emission angle, the arrival direction of the photon with respect to the photomultiplier tube (PMT), and the distance of the shower vertex to the PMT. PMTs that count zero photons are also considered in the Poisson likelihood. The likelihood used in the direction fit is:

$$
\begin{aligned}
\mathscr{L}= & \sum_{i=1}^{N_{\text {selected Hits }}} \log \left\{P_{q>0}\left(q_{i} \mid E_{v}, d_{i}, \phi_{i}, \alpha_{i}\right)+P_{\mathrm{bg}}\left(q_{i}\right)\right\} \\
& +\sum_{i=1}^{N_{\text {unhit PMTs }}} \log \left\{P_{q=0}\left(E_{v}, d_{i}, \phi_{i}\right)\right\}
\end{aligned}
$$

with:

$q_{i}$, the charge of hit $i$,

$P_{q>0}$, the probability for a hit PMT to measure its observed charge,

$P_{q=0}$, the probability for a PMT to not being hit,

$P_{\mathrm{bg}}$, the probability for that hit to be caused by random background,

$E_{v}$, the neutrino energy,

$d_{i}=\left|\vec{r}_{\mathrm{PMT}, i}-\vec{r}_{\text {shower }}\right|$, the distance between the shower mean and PMT $i$,

$\phi_{i}$, the angle between $\left(\vec{r}_{\mathrm{PMT}, i}-\vec{r}_{\text {shower }}\right)$ and the neutrino direction,

$\alpha_{i}$, the angle between $\left(\vec{r}_{\mathrm{PMT}, i}-\vec{r}_{\text {shower }}\right)$ and the direction the PMT is facing,

$\vec{r}_{\text {shower }}$, the position of the shower mean.

\footnotetext{
${ }^{1}$ The M-estimator is a modified $\chi^{2}$-test that is less sensitive to outliers: $M_{\mathrm{est}}=2 \cdot \sqrt{1+\chi^{2} / 2}-2$.
} 
The shower position can be reconstructed very reliably. Figure (1) shows the longitudinal (left) and perpendicular (right) offset of the position fit with respect to the Monte Carlo neutrino axis. For electromagnetic showers (red data points), the reconstructed position along the shower axis corresponds to the mean of the shower's light emission spectrum (purple line in the figure). Hadronic showers (blue data points) have a different emission profile and are usually reconstructed a bit further along the shower axis. The feature in the em-shower channel just below $E_{V}=10^{7} \mathrm{GeV}$ is due to the Glashow-Resonance. Here, an anti electron neutrino interacts with an electron from the ambient water and produces a $W^{-}$Boson. If this $W^{-}$decays hadronically, it produces a hadronic shower that carries the whole energy of the original neutrino (in contrast to neutral current interactions where the hadronic shower only takes a fraction of the neutrino energy). The observed longitudinal offset, therefore, corresponds to a high energetic hadronic shower and is expected to lie further away than the ones for pure em-showers. The median perpendicular distance to the neutrino axis is as low as $1 \mathrm{~m}$ in either case over a wide energy range.
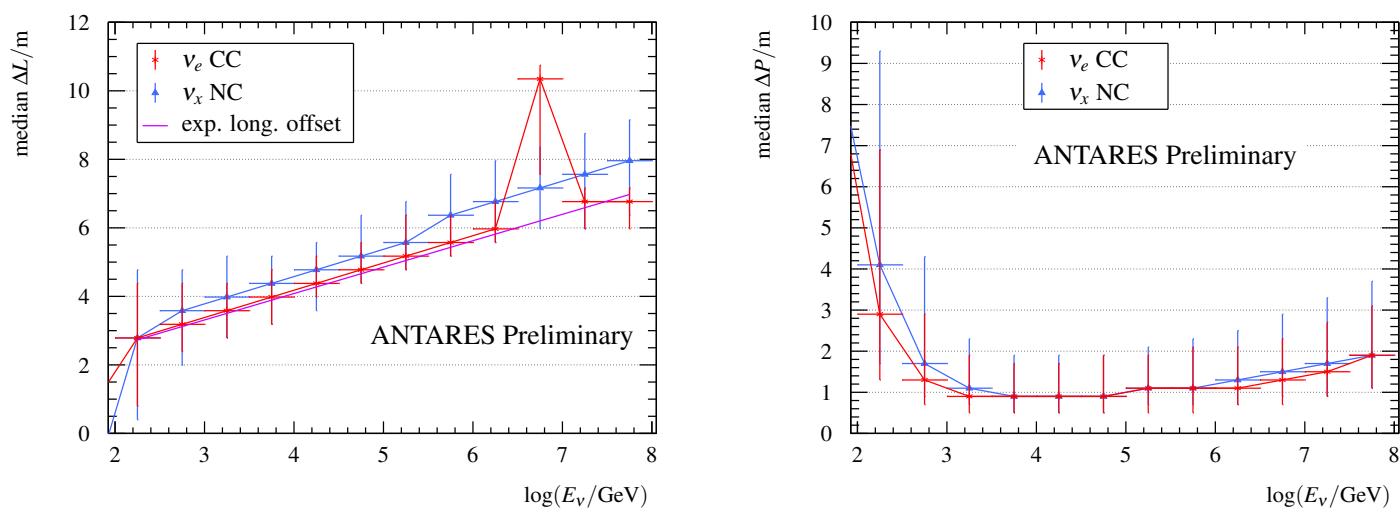

Figure 1: Performance of the shower position reconstruction, red for electromagnetic showers, blue for hadronic showers, both after containment and error estimator cut (see section 2.1), the purple line is the mean of the light emission spectrum for em-showers - Left: The distance between the position of the neutrino interaction vertex and the reconstructed shower position along the neutrino axis. Right: The distance of the reconstructed shower position perpendicular to the neutrino axis.

The angular resolution of the shower reconstruction is highly energy dependent. For energies $10^{4} \lesssim E / \mathrm{GeV} \lesssim 10^{6}$ it reaches median resolutions as low as $3^{\circ}$ with a $1 \sigma$ lower spread below $2^{\circ}$. Below this energy range, not enough light is produced to illuminate sufficient PMTs for a proper reconstruction and above, most of the PMTs are saturated and the limited size of the ANTARES detector prevents us from accessing higher energies with proper resolutions. While not presently used in the point source search, it is worth mentioning that the statistical energy resolution of about $5 \%$ has been achieved. A systematic underestimation of about $20 \%$ can be observed over the whole energy range which is easily corrected post-reconstruction. See figure (2) for the performance of the direction (left) and energy (right) reconstruction.

The angular resolution of the cascade reconstruction can also be measured in data using a sample of atmospheric muons which also have a reconstructed cascade. If the reconstructed cascade corresponds to a true EM-shower which originates from the stochastic muon energy loss, the shower will have the same direction as the muon to a good approximation. As the muon is accurately reconstructed by the track fit, a sample of EM cascades of known direction can be isolated. 

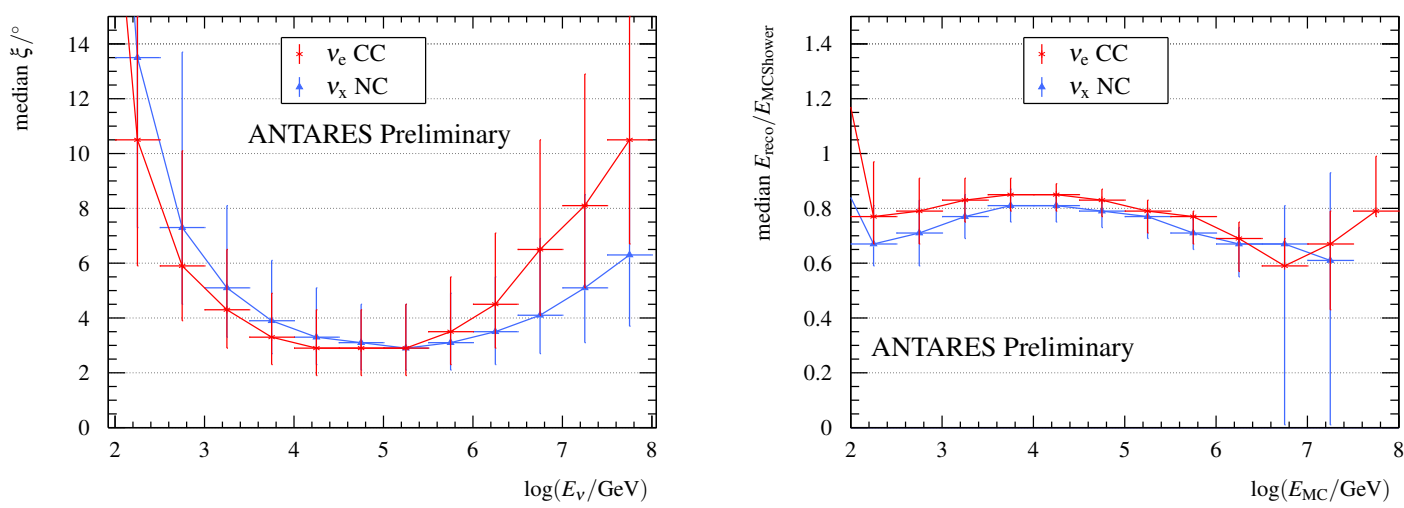

Figure 2: Performance of the shower energy-direction reconstruction, red for electromagnetic showers, blue for hadronic showers, both after containment and error estimator cut (see section 2.1) - Left: The angle between the directions of the reconstructed shower and the Monte Carlo neutrino. Right: The ratio between the reconstructed energy and the Monte Carlo shower energy.

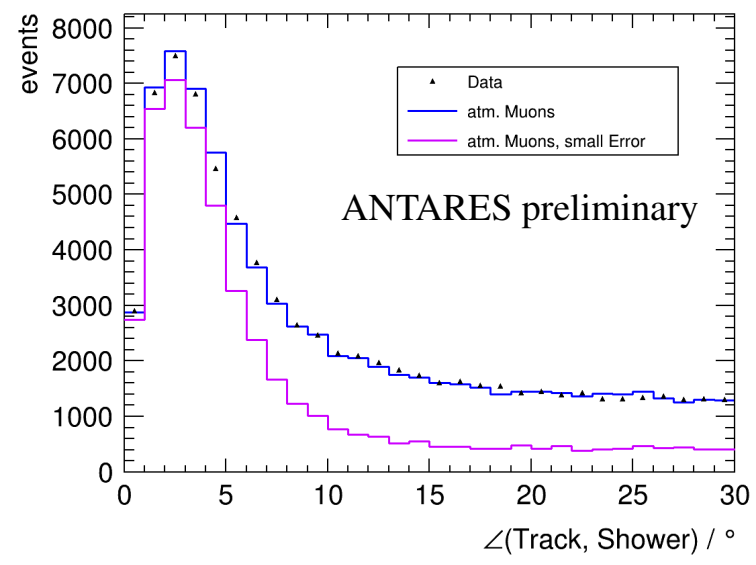

Figure 3: The angular separation of the reconstructed directions using track and shower hypotheses applied to the same atmospheric muon events - black: data, blue: atmospheric muons, violet: muons with a track reconstructed better than $5^{\circ}$.

Figure (3) shows the result for a loose selection. A clear population of well reconstructed showers is visible; with a resolution of two to three degrees (maximum of the distribution). This peak is well modelled in simulations of atmospheric muons[2], which implies the Monte Carlo can be reliably used to determine the resolution for cascades of cosmic origin.

The median energy-integrated angular resolution for an $E^{-2}$ charged-current $v_{e}$ signal for the selection adopted in the analysis is 3 degrees.

A very similar shower reconstruction algorithm is being used for KM3NeT, which also achieves angular resolutions of $\mathscr{O}\left(1^{\circ}\right)$.

\subsection{Selection and data sample}

The selection of tracks (i.e. $v_{\mu}$ candidates) is identical to [1]. It requires tracks to be up-going $(\cos (\vartheta)>-0.1)$, with a small estimated angular error $\left(\beta<1^{\circ}\right)$ and with a minimum reconstruction quality parameter $(\Lambda>-5.2)$.

Cascade candidates are selected using a set of criteria aimed at rejecting background from atmospheric muons, which are misreconstructed as up-going cascades - too many to describe them 
all in detail here. The selection requires:

- the event not to be selected by the track channel,

- reconstructed as up-going $(\cos (\vartheta)>-0.1)$

- the shower position to be close to the detector $(\rho<300 \mathrm{~m},|z|<250 \mathrm{~m})$,

- a maximal angular error estimate $\left(<10^{\circ}\right)$,

- passing a combined cut on the GridFit Ratio[3] and number of selected hits,

- passing a muon/em-shower likelihood discrimination specifically developed for this analysis,

- a sufficiently low ratio between "early" and "on-time" charge

The used data period from 2007 to 2013 with a life time of 1622 days contains 6261 muon track candidates, $10 \%$ of which is estimated to be atmospheric muons. A total of 156 cascade events are selected; this sample is estimated to consist to $90 \%$ of atmospheric neutrinos, while the rest are atmospheric muons.

For an $E^{-2}$ signal flux with 1:1:1 flavour composition, the selected cascade events are expected to increase in signal event rate by $30 \%$.

\section{Search method}

The signature of a point source is a cluster of events. The distribution of the angle of deviation between the reconstructed signal event and the location of the source is described by the point spread function $\mathscr{F}(\gamma)$, which is the probability density of reconstructing an event at an angular distance $\gamma$ from the true source. In order to distinguish this signature from random clusters of background events, we use a likelihood ratio. It is convenient to express the intensity of the source in terms of the mean number of detected events that the source produces: $\mu_{\text {sig }}$. The likelihood of the data is given by:

$$
\log \mathscr{L}_{\text {s } \mathrm{b}}=\sum_{i} \log \left[\mu_{\text {sig }} \times \mathscr{F}\left(\gamma_{i}\right) \times \mathscr{N}_{\text {sig }}\left(N_{\mathrm{i}}^{\text {Hits }}\right)+\mathscr{B}\left(\delta_{\mathrm{i}}\right) \times \mathscr{N}_{\text {backg }}\left(N_{\mathrm{i}}^{\text {Hits }}\right)\right]-\mu_{\text {tot }},
$$

where $\gamma_{i}$ is the angle between the reconstructed direction and the assumed source coordinates. $\mathscr{N}$ is the distribution for the number of selected hits for the signal / background case. $\mathscr{B}_{i}$ is the rate of background events at the coordinates of event $i$. For simplicity, we consider the background rate to be a function of declination. The term $\mu_{\text {tot }}$ represents the total number of expected events. The sum in the likelihood takes muon track as well as shower events into account and uses the proper ingredients for $\mathscr{F}_{i}$ and $\mathscr{B}_{i}$. Since events that are very far away from the source position yield a constant contribution and will not influence maximum likelihood estimates, the sum can be restricted to a reasonably small cluster of events around the hypothesized source position. An analogous argument allows replacing $\mu_{\text {tot }}$ with $\mu_{\text {sig }}$ in equation (3.1).

The first step to compute the likelihood ratio is to fit the three free parameters $\left(\mu_{\mathrm{sig}}, \delta_{s}, \alpha_{s}\right)$ in the signal hypothesis to the cluster. In case of a fixed-point search, the coordinates are fixed and the fit has only $\mu_{\text {sig }}$ as a free parameter. A selection of IceCube muon candidates has been adopted as point source candidates[4]. Since those events have angular error estimators between one and two degrees, we do not treat them like the usual point source candidates with a fixed position but also fit the direction within a cone of $2^{\circ}$. 
Finally, to distinguish signal-like clusters from clusters produced by background, we compute the likelihood ratio $Q$ :

$$
Q=\log \mathscr{L}_{\mathrm{s}+\mathrm{b}}^{\max }-\log \mathscr{L}_{\mathrm{b}},
$$

where the first term is the likelihood evaluated for the best-fit parameters and the second term is equation (3.1) evaluated for $\mu_{\text {sig }}=0$. As we will use $Q$ to differentiate between signal and background, it is also called the test statistic.

\section{Sensitivity and discovery potential}

The detector sensitivity and discovery potential can be determined with pseudo experiments. For this, various numbers of signal events are injected at a fixed position (distributed according to the point spread function $\mathscr{F}$ ) on top of a background as found in real data.

\subsection{Full sky search}

Figure (4a) shows the fitted right ascension for various numbers of injected signal at a fixed position in the sky $\left(\alpha=100^{\circ}, \delta=-70^{\circ}\right)$. Figure (4b) shows the flux needed in a full sky search to have a $5 \sigma$ discovery in $50 \%$ of hypothetical, equivalent experiments.

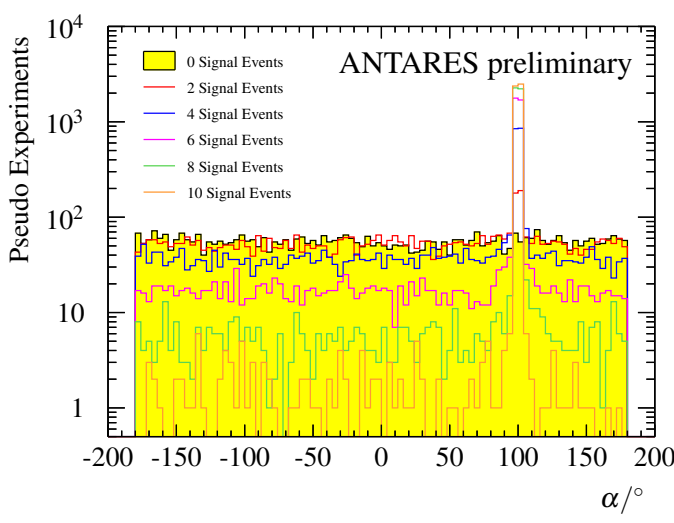

(a) fitted right ascension for pseudo experiments

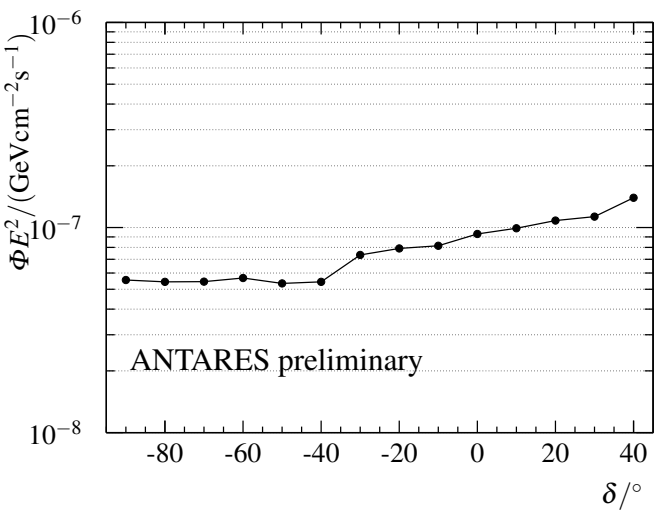

(b) discovery flux for full sky search

Figure 4: (a) Fitted right ascension for pseudo experiments for various numbers of signal events injected at $\delta=-70^{\circ}$ and $\alpha=100^{\circ}$. (b) The flux necessary for a $50 \%$ probability for a $5 \sigma$ discovery in a full sky search.

\subsection{Candidate List Search}

Figure (5a) shows the number of signal event found by the likelihood fit for different numbers of injected signal events. The fit tends to slightly overestimate the amount of injected signal by about half an event. Figure (5b) shows the flux needed in a candidate list search to have a $5 \sigma$ discovery in $50 \%$ of hypothetical, equivalent experiments. Figure (5c) shows the flux that can be excluded with a confidence level of $90 \%$ in case no signal events could be found. The expected sensitivity for the fixed point search is $10^{-8} \cdot \mathrm{E}^{2} \mathrm{GeVcm}^{-2} \mathrm{~s}^{-1}$ for declinations below $-40^{\circ}$. 


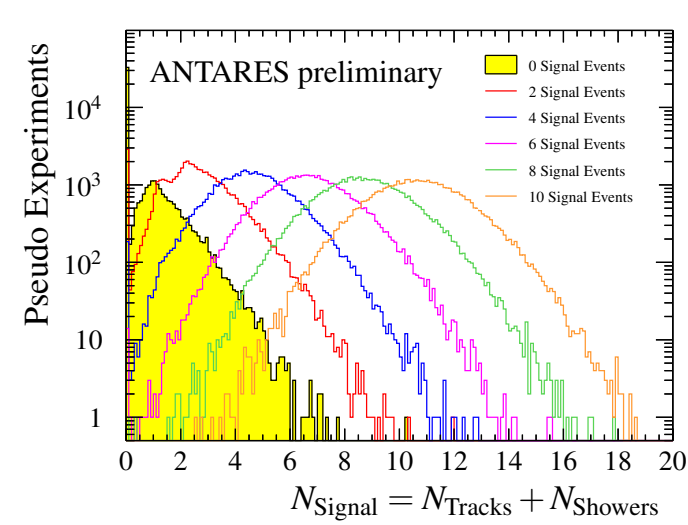

(a) number of fitted signal events

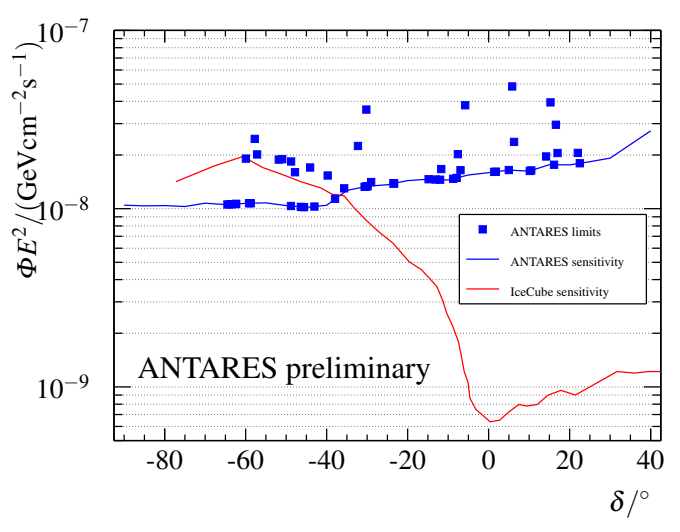

(c) sensitivity for the fixed search

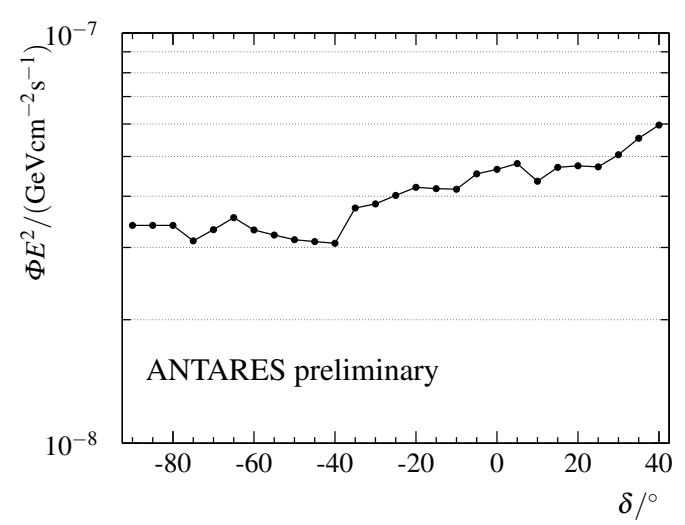

(b) discovery flux for fixed search

Figure 5: (a) The number of fitted events (Tracks + Showers) for different numbers of injected signal at $\delta=-70^{\circ}$ and $\alpha=100^{\circ}-$ (b) The flux needed to claim a $5 \sigma$ discovery in $50 \%$ of the cases - (c) The sensitivity for the fixed point search: blue for ANTARES, red for IceCube as comparison.

\section{Results}

No discovery can be claimed - neither in the full sky nor in the candidate list search. The following subsections show the significances for the different search methods - figure (6) shows their respective most significant clusters.

\subsection{Full Sky Search}

The most significant cluster in the full sky search is very close to the one in the previous trackonly analysis. It is located at $\alpha=-48.3^{\circ}, \delta=-64.6^{\circ}$ (old track-only analysis: $\alpha=-46^{\circ}, \delta=$ $-65^{\circ}$ ). Within $3^{\circ} 16$ tracks were found and 1 shower within $10^{\circ}$. The fitted number of signal events is $N_{\mathrm{Sig}}=5.5+0.8$ (Tracks + Showers). The measured p-value is $18.5 \%$ which corresponds to a significance of $1.33 \sigma$. In the previous analysis, this cluster had a significance of $2.17 \sigma$ which suggests that adding the shower channel exposes this cluster as a mere over-fluctuation in the track channel.

\subsection{Candidate List Search}

The cluster with the highest significance in the candidate list search is HESSJ0632+057 $\left(\alpha_{\mathrm{s}}=\right.$ $\left.98.24^{\circ}, \delta_{\mathrm{s}}=5.81^{\circ}\right)-$ the same source as in the last analysis using only tracks. With 36 tracks and 
0 showers within $10^{\circ}$ around the source, the fit found $N_{\mathrm{Sig}}=1.2+0.2$ (Tracks + Showers) signal events, corresponding to a significance of $0.75 \sigma$.

\subsection{IceCube Candidate Search}

The IceCube muon track candidate with the highest significance is the event with the IceCube ID $28\left(\alpha_{\mathrm{IC}}=164.8, \delta_{\mathrm{IC}}=-71.5, \beta_{\mathrm{IC}}=1.3^{2}\right) .7$ tracks have been found within $3^{\circ}$ and 0 showers within $10^{\circ}$. The fitted signal is $N_{\mathrm{Sig}}=0.005+0.001$ with a significance of $0 \sigma$.

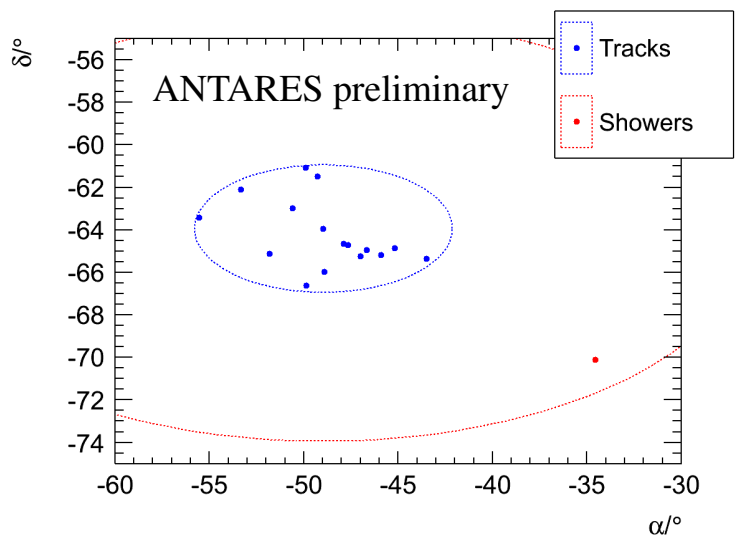

(a) full sky search

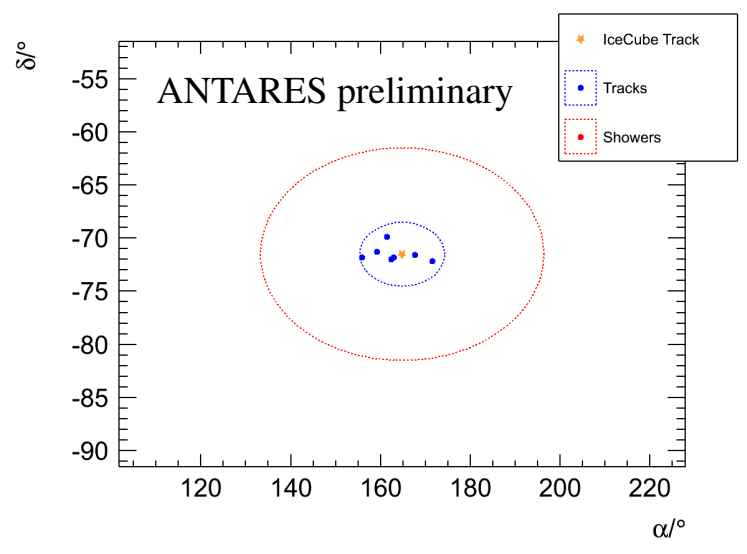

(c) IceCube candidate search

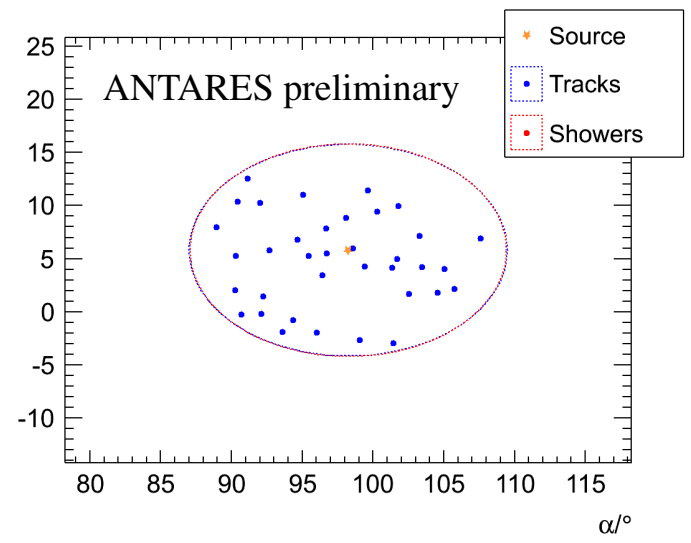

(b) candidate list search

Figure 6: Most significant clusters for different search methods: (a) full sky search (b) candidate list search (c) IceCube candidate search. The dashed lines mark the range in which events are considered for the current cluster.

\section{References}

[1] S. Adrián-Martínez et al., 2014, ApJ 786 L5, doi:10.1088/2041-8205/786/1/L5

[2] Y. Becherini et al., A parametrisation of single and multiple muons in the deep water or ice, Astrop. Phys. 25, 1, (2006)

[3] E. Visser, Neutrinos from the Milky Way, PhD Thesis, (2015)

[4] The IceCube Collaboration, Phys. Rev. Lett. 113, 101101 (2014)

[5] M. G. Aartsen et al., 2014 ApJ 796109

\footnotetext{
${ }^{2} \beta \rightarrow$ angular error estimate
} 